\title{
Incubation time after pulsed electric field treatment of microalgae enhances the efficiency of extraction processes and enables the reduction of specific treatment energy
}

Aude Silve*, Chua Boon Kian, loannis Papachristou, Christin Kubisch, Natalja Nazarova, Rüdiger

Wüstner, Klaus Leber, Ralf Strässner, Wolfgang Frey

Karlsruhe Institute of Technology, Institute for Pulsed Power and Microwave Technology (IHM),

Eggenstein-Leopoldshafen, Germany

*Corresponding author:

Dr. Aude Silve,

Hermann-von-Helmholtz-Platz 1, Bldg 630

76344 Eggenstein-Leopoldshafen,

Germany,

aude.silve@kit.edu

+4972160823936 


\begin{abstract}
:
Pulsed Electric Field (PEF) pre-treatment, applied on fresh microalgae Auxenochlorella protothecoides, induces spontaneous release of a substantial water fraction and enables subsequent lipid extraction using ethanol-hexane blends. In this study, fresh microalgae suspensions were treated with PEF and incubated under inert conditions. Incubation promotes the release of ions and carbohydrates and increases the yields of subsequent lipid extraction thus enabling a considerable reduction of PEF-treatment energy. With a 20 hour incubation period at $25^{\circ} \mathrm{C}$, almost total lipid extraction is achieved with a specific PEF-treatment energy of only $0.25 \mathrm{MJ} / \mathrm{kg}_{\text {Dw. }}$. Incubation on ice remains beneficial but less efficient than at $25^{\circ} \mathrm{C}$. Additionally, incubating microalgae cells in suspension at $100 \mathrm{~g}_{\mathrm{DW}} / \mathrm{L}$ or in a dense paste, was almost equally efficient. Correlation between the different results suggests that spontaneous release of ions and carbohydrates facilitates more successful lipid extraction. A direct causality between the two phenomena remains to be demonstrated.
\end{abstract}

\title{
Keywords:
}

Microalgae; Lipid; Biofuel; PEF treatment; Electroporation; Electropermeabilisation 


\section{Introduction}

Microalgae's rich intracellular content is attractive for many applications such as fuel, food or feed. Nevertheless, only a few microalgae derived products have reached the market. Indeed, both, the production and the downstream-processing of large biomass quantities are not efficient enough for most products to be profitable yet (Posten and Walter, 2012). The current trend is to develop microalgae bio-refinery in order to achieve profitability through multi-components valorization (Chew et al., 2017; Günerken et al., 2015; Vanthoor-Koopmans et al., 2013). Downstream processing should operate on wet biomass basis to avoid drying costs (Cooney et al., 2011), should enable efficient extraction of intracellular compounds and additionally should enable cascade processing. Success of such a process highly relies on the pre-treatment of the biomass regardless of whether it is performed by biological, chemical or physical means (Günerken et al., 2015). Among the physical pre-treatment methods, one can distinguish between mechanical methods aiming at complete disruption of the cells such as bead-milling (BM) or high pressure homogenization $(\mathrm{HPH})$, and other approaches which affect the integrity of cells without reducing them into debris. The first approach is reducing microalgae biomass to a mixture of small cell fragments in which intracellular components are spread and in principle accessible for recovery. These approaches are very promising for some applications. HPH in particular has already reached a high technology readiness level. Nevertheless, the creation of very small debris and in some cases even formation of stable emulsions is considered a serious drawback for all applications requiring cascade processing of the biomass and in particular separation of all solid debris from the liquid phase. Additionally, albeit those methods are not intrinsically thermal based, the intense mechanical forces at play, e.g. high shear stresses, induce high local temperature increases which can damage thermo-sensitive molecules (Phong et al., 2018). Finally, these methods aim at 
mechanically disrupting the cell-wall of microalgae which can represent an additional challenge for those approaches when pre-treatment is performed on one of the many very robust microalgae strain. Optimization of HPH and BM has nevertheless enabled considerable reduction of energy demand. A straight comparison of the energy requested by different disruption technique is a difficult task since each individual study relies on a specific and individual diagnostic. As a guide, one can still note that in the current state of the art, the lowest reported values for BM are in the range of 1.6 to $3.6 \mathrm{MJ} / \mathrm{kg}_{\mathrm{DW}}$ (Postma et al., 2017, 2015). In the case of HPH, values as low as $0.16 \mathrm{MJ} / \mathrm{kg}_{\mathrm{DW}}$ have been reported for weak species, while resistant species, such as Nannochloropsis, still require at least around $3.4 \mathrm{MJ} / \mathrm{kg}_{\mathrm{DW}}$ when treated directly after harvesting. For the latter, pre-incubation for $15 \mathrm{~h}$ at $37^{\circ} \mathrm{C}$ was shown to significantly weaken cell wall and enable efficient disruption with only 0.4 MJ/kg Dw (Olmstead et al., 2013; Spiden et al., 2013; Yap et al., 2015).

Other physical pre-treatment methods, whether mechanical such as ultrasounds, thermal such as autoclaving, or electrical such as microwaves and pulsed electric field (PEF), rely on a different principle since, according to the currently published literature, they do not lead to a distinct disruption of microalgae cells in the sense that cells are not fragmented into debris. The integrity of cells is however compromised which can facilitate further extraction of intracellular valuables (Grimi et al., 2014; Günerken et al., 2015). In most cases, the underlying mechanisms are not described or only partially explained in literature. In the specific case of PEF, more is known about the mechanisms since the effect of pulsed electric fields on biological cells has been intensively studied in other scientific communities (Pakhomov et al., 2010; Teissie et al., 2005). As the electric field is established in the cells surrounding, charging of cell plasma membrane occurs, resulting in high transmembrane voltage and in turn loss of integrity of the membrane with increase of its 
conductivity and its permeability. This phenomenon, known as electroporation or electropermeabilisation, has been intensively studied but the exact underlying molecular mechanisms still remain to be explained. Nevertheless, it is admitted that the lipid bilayer part of the cell membrane is the structure which is affected. The creation of hydrophilic pores in the lipid bilayer is strongly supported by theoretical approaches (Poignard et al., 2016) as well as by molecular dynamics simulations (Levine and Vernier, 2010; Tarek, 2005) but experimental evidences of pores are still lacking. A direct visualization of pore-like structures in some artificial bilayers was published recently (Sengel and Wallace, 2016) but a comprehensive description of electroporation at cell level appears to be more complex. Despite these remaining open questions, PEF-treatment has already been successfully implemented for clinical applications (Mir, 2008, 2006; Yarmush et al., 2014) as well as for industrial applications in the food-industry (Frey et al., 2017; Toepfl et al., 2006).

Since a couple of years PEF-treatment is considered and evaluated as a pre-treatment method for microalgae (Kempkes, 2016; Golberg et al., 2016; Frey et al., 2017). Several studies have already detected PEF-induced electropermeabilisation of microalgae cells, using uptake of markers such as Calcein (Azencott et al., 2007), Nile-red (Silve et al., 2018), and propidium iodide (Bodénès et al., 2016; Luengo et al., 2015) or release of molecules such as ions or carbohydrate (Goettel et al., 2013) as a central diagnostic approach. Uptake of large permeabilisation markers such as bovine serum albumin appears to be less efficient (Azencott et al., 2007), which is a general trend observed on mammalian cell studies but which might be even reinforced by the presence of the cell-wall when focusing on plant cells. In terms of microalgae processing for bio-refinery, it was already demonstrated that PEF could induce spontaneous release of some water soluble molecules, including some carbohydrates (Carullo et al., 2018; Goettel et al., 2013; Postma et al., 
2016), some water soluble proteins (Carullo et al., 2018; Coustets et al., 2015, 2013; Goettel et al., 2013; Postma et al., 2016) and pigments (Luengo et al., 2015). Additionally, PEF-treatment or assimilated approaches (Sheng et al., 2011) have been shown to facilitate solvent extraction of lipids (Silve et al., 2018; Zbinden et al., 2013; Eing et al., 2013; Sheng et al., 2011; Parniakov et al., 2015; Grimi et al., 2014; Lai et al., 2014).

The energy required for efficient PEF pre-treatment of microalgae is still under investigation. In general, PEF-treatment energy scales with the conductivity of the suspension to be treated. To avoid energy-intensive and water-consuming washing steps, PEF-treatment at native suspension conductivity appears to be advantageous. The lowest reported values for PEF treatment of unwashed microalgae suspensions are typically in the range of 1.5 to $2 \mathrm{MJ} / \mathrm{kg}_{\mathrm{DW}}$ (Goettel et al., 2013; Postma et al., 2016). In cell wall deficient C. reinhardtii, and with additional washing of microalgae suspensions, energy values as low as $180 \mathrm{~kJ} / \mathrm{kg}_{\mathrm{DW}}$ were also reported to be efficient for extraction of proteins ('t Lam et al., 2017). Special emphasis on energy efficiency of PEF treatment is essential for applications focusing on low added-value products recovery or more in general for applications requesting processing of large amounts of biomass. This is in particular valid for biodiesel production, for which it is essential, that the specific energy demand of the pretreatment remains as low as possible in order to maintain low overall processing costs.

In case of PEF treatment performed in batch mode, the specific treatment energy $\mathrm{W}\left[\mathrm{J} / \mathrm{kg}_{\mathrm{DW}}\right]$ is given by (1) and is a function of the conductivity of the suspension $\sigma[\mathrm{S} / \mathrm{m}]$, of the electric field intensity $E[V / m]$, of the duration of the pulses $\Delta t[s]$, of the number of pulses applied to the suspension $\mathrm{N}$ and of the concentration of the biomass $\mathrm{C}[\mathrm{kg} \mathrm{DW} / \mathrm{L}]$. 


$$
W=E^{2} \cdot \sigma \cdot \frac{\Delta t \cdot N}{C}
$$

In case of a continuous flow process as depicted in Fig. 1, the number of pulses $\mathrm{N}$ applied on a given volume unit is a function of the repetition rate $f_{\text {rep }}[\mathrm{Hz}]$ and of the residence time $t_{\text {res }}[s]$ inside the treatment chamber i.e. between the two electrodes.

$$
N=f_{\text {rep }} \cdot t_{\text {res }}
$$

In first approximation, if the fluid velocity $\mathrm{v}_{\mathrm{fl}}[\mathrm{m} / \mathrm{s}]$ is considered to be homogeneous, then the residency time $t_{\text {res }}$ for any volume unit is given by the length of the treatment chamber $L[m]$ divided by the fluid velocity.

$$
t_{r e s}=\frac{\mathrm{L}}{v_{f l}}
$$

Finally, the fluid velocity $\mathrm{v}_{\mathrm{fl}}$ can be expressed as a function of the flow rate $Q\left[\mathrm{~m}^{3} / \mathrm{s}\right]$ and of the cross section of the treatment chamber w.d $\left[\mathrm{m}^{2}\right]$ as follows:

$$
v_{f l}=\frac{\mathrm{Q}}{w \cdot d}
$$

In that case, the specific treatment energy $W$ is given by the equation:

$$
W=E^{2} \cdot \sigma \cdot L \cdot w \cdot d \cdot \frac{\Delta t . f_{r e p}}{Q \cdot C}
$$

For given treatment chamber dimensions, several strategies for reducing the specific PEFtreatment energy demand can be considered. The first strategy would be to reduce the conductivity of the microalgae suspension. This can be easily done by washing the biomass with distilled water. As already hinted introductorily above, washing itself would induce additional energy costs and increase the water consumption which might counter balance the benefit of 
energy reduction for the PEF treatment processing step. Therefore, such a strategy should be restricted to sea-water microalgae suspensions, exhibiting very high initial conductivities.

Another approach would be to increase the biomass concentration of the treated suspension since experiments on organic carbon release have shown that the efficiency of the PEF-treatment does not depend on the biomass concentration (Goettel et al., 2013). A net energy consumption reduction can therefore be obtained, as long as it is possible to pump the biomass through the treatment chamber and as long as the energy costs due to concentration remain low enough. Reduction of energy consumption can also be achieved by modifying the intrinsic pulse parameters of the PEF-treatment i.e. by reducing the electric field or the pulse duration, although this is likely to reduce the efficiency of the permeabilization. Finally, it is possible to increase the flow rate $Q$ or to reduce the repetition rate $f_{\text {rep }}$, which are both equivalent to reducing the number of pulses delivered per volume unit, a strategy also likely to reduce the efficiency of the PEFtreatment.

In previous studies, PEF-treatment applied on the microalgae Auxenochlorella protothecoides induced spontaneous release of a water fraction representing more than $10 \%$ of the biomass, mostly in the form of carbohydrates but also in the form of ions or proteins (Goettel et al., 2013). Additionally, PEF-treatment applied prior to lipid extraction with Ethanol-Hexane blends, enabled to reach almost total lipid extraction while yields from non-treated biomass were close to zero (Silve et al., 2018). Most experiments were performed with a fixed energy input of $150 \mathrm{~kJ} / \mathrm{L}$ and a biomass concentration of $100 \mathrm{~g} / \mathrm{L}$ i.e. $10 \% \mathrm{w} / \mathrm{v}$ therefore translating into $1.5 \mathrm{MJ} / \mathrm{kg}_{\mathrm{DW}}$. The main target of this study was to investigate to what extent energy could be reduced without affecting the spontaneous release of the water-fraction products and without impacting the lipid-extraction yield. Energy was reduced by decreasing the repetition rate of the pulses and therefore the 
average number of pulses administrated to a suspension volume-unit. All other parameters were kept constant.

Moreover, several studies in the literature suggested that an incubation period after PEFtreatment could increase the amount of water-soluble molecules released into the interstitial medium (Coustets et al., 2015, 2013; Goettel et al., 2013). Indeed, It is assumed that this release after PEF-treatment is partially or fully driven by diffusion across the permeabilized membrane. In the case of microalgae cells, these molecules need to diffuse across a very small distance, typically the dimension of the cell i.e. a few micrometers. Free diffusion cannot take place as both the cell membrane (albeit permeabilized) and the cell wall (most likely unaffected by the PEF treatment) impose restriction on migration of water-soluble molecules into the interstitial medium. Therefore, the required time for diffusion cannot be predicted easily and can vary with the treatment energy. It has been also suggested that an incubation after PEF could facilitate further solvent extraction (Parniakov et al., 2015) despite the fact that no spontaneous release of lipids was observed. Based on the above considerations, this study included a systematic investigation of the effect of incubation duration after PEF-treatment on the release of carbohydrates, ions or charged molecules, and on lipid yields obtained from solvent extraction.

\section{Material and Methods}

\subsection{Microalgae Cultivation and Harvesting}

All experiments were performed with Auxenochlorella protothecoides (A.p.), strain number 211-7a obtained from SAG, Culture Collection of algae, Göttingen, Germany. Axenic cultures were maintained in glass cultivation flasks in a modified Wu medium (detailed composition can be 
found in (Silve et al., 2018)). Experiments were performed on microalgae cultivated either mixotrophically in cultivation flasks containing glucose as a carbon source or autotrophically in $25 \mathrm{~L}$ photo-bioreactors bubbled with air enriched at $3 \%$ of $\mathrm{CO}_{2}$ as the only carbon source. The details of cultivation including medium composition can be found in (Silve et al., 2018). After harvesting, the microalgae suspension was concentrated by centrifugation at $3000 \mathrm{~g}$ using a Sigma 8k centrifuge (Sigma Laborzentrifugen $\mathrm{GmbH}$, Osterode am Harz, Germany), with a swinging-bucket rotor. The microalgae pellet was resuspended in the appropriate amount of cultivation medium in order to obtain the desired concentration of $100 \mathrm{~g} \mathrm{DW} / \mathrm{L}$. The process therefore did not include any washing step. The exact final concentration was always measured. The average delay, induced by the step of concentration of the microalgae, from the beginning of the harvest to the beginning of the PEF experiment, was typically $2 \mathrm{~h}$. For all experiments, a fraction of the final suspension was freezedried and stored in vacuumed sealed bags at $-20^{\circ} \mathrm{C}$ for further analysis of the biomass.

\subsection{PEF Treatment}

The concentrated microalgae suspension was treated in continuous mode with a constant flow rate of $\mathrm{Q}=0.1 \mathrm{~mL} / \mathrm{s}$. The treatment chamber consisted of two parallel circular stainless steel electrodes separated by a polycarbonate housing. Distance between the electrodes was $4 \mathrm{~mm}$. The setup ensured a uniform electric field distribution in the whole volume of the treatment chamber $\left(\mathrm{V}_{\text {chamber }}=48 \times 11 \times 4 \mathrm{~mm}^{3}\right)$ which had no sharp angles. Photos of the treatment chamber and of the electrodes can be found in (Goettel et al., 2013). PEF treatment was performed with a custom-made transmission-line generator. Pulse duration, was fixed at $\Delta t=1 \mu \mathrm{s}$, electric field intensity at $E=4 \mathrm{MV} / \mathrm{m}$. The pulse repetition rate $\mathrm{f}_{\text {rep }}$ was adjusted between $0.1 \mathrm{~Hz}$ and $3 \mathrm{~Hz}$, in order to adjust the specific treatment energy as described in table 1. More details about the instrumentation and the energy calculation were already given in (Silve et al., 2018). In 
experiments comparing the effect of different specific treatment energies, $12 \mathrm{~mL}$ of suspension were processed at each given energy setting. The total treatment duration was 2 min per sample. The maximum increase of temperature $\Delta \mathrm{T}_{\max }\left[{ }^{\circ} \mathrm{C}\right]$ of the microalgae samples due to deposition of the PEF energy are reported in the last column of table 1 and were calculated assuming adiabatic conditions (Eq. 6) and using the specific heat capacity $c_{p}[\mathrm{~J} / \mathrm{K} / \mathrm{kg}]$ and the density $\rho[\mathrm{kg} / \mathrm{L}]$ of water.

$$
\Delta T_{\max }=\frac{W_{\text {sus }}}{c_{p} \rho}
$$

\subsection{Incubation of samples for extraction experiment}

In all extraction experiments, samples were either processed immediately after PEF-treatment or after a given time of incubation. For incubation, samples were flushed with nitrogen and kept in the dark either at $25^{\circ} \mathrm{C}$ or on ice. Temperature of samples on ice was between 1 and $3^{\circ} \mathrm{C}$. After incubation, the samples were centrifuged for $5 \mathrm{~min}$ at $10000 \mathrm{~g}$. After measurement of its conductivity, the supernatant was stored at $-20^{\circ} \mathrm{C}$ for further analysis of carbohydrate content. Lipid extraction was then performed on the microalgae pellet. In some experiments, the centrifugation was applied directly after PEF-treatment and only the dense microalgae pellet was incubated. In that case, the pellet was also flushed with nitrogen and placed in the dark.

\subsection{Conductivity measurements}

The conductivity $\sigma[\mu \mathrm{S} / \mathrm{cm}]$ of the microalgae suspensions and of microalgae supernatants was measured using a conductivity meter (Endress + Hauser, CLM 381). No automatic temperature compensation was used, but temperature $\mathrm{T}\left[{ }^{\circ} \mathrm{C}\right]$ was recorded in parallel with conductivity. The equivalent conductivity at $25^{\circ} \mathrm{C}, \sigma_{25}[\mu \mathrm{S} / \mathrm{cm}]$, was calculated using Eq. 7 where $\alpha_{25}$ is the 
temperature coefficient of variation at $25^{\circ} \mathrm{C}$ (Grimnes and Martinsen, 2008). The coefficient $\alpha_{25}$ was obtained experimentally by measuring conductivity of a microalgae suspension at different temperature (data not shown) and had a value of $2.8 \% /{ }^{\circ} \mathrm{C}$.

$$
\sigma_{25}=\sigma_{T} \frac{1}{1+\alpha_{25}(T-25)}
$$

\subsection{Carbohydrate measurements}

Determination of carbohydrate release into the supernatant after PEF treatment was performed using the Anthrone Sulfuric Acid assay. Fresh starch aqueous solutions with concentrations ranging from $0.02 \mathrm{~g} / \mathrm{L}$ to $0.4 \mathrm{~g} / \mathrm{L}$ were prepared from starch powder (Merck 1.01257). They were used as standards and processed like the samples. The frozen supernatants collected after PEF treatment were thawed, diluted in distilled water at the appropriate concentration and kept on ice. For absolute determination of carbohydrate content, freeze-dried biomass was resuspended in distilled water and diluted to a concentration ranging between 0.1 and $0.4 \mathrm{~g} / \mathrm{L}$. All samples were processed in triplicates. The anthrone reagent was prepared on the day of the experiment by dissolving anthrone (Merk 1.01468) in 95\% sulfuric acid (AnalaR NORMAPUR: VWR Chemicals 20700) at a final concentration of $0.1 \% \mathrm{w} / \mathrm{v}$. The solution was well mixed and kept on ice for at least 5 minutes. Afterwards $400 \mu \mathrm{L}$ of diluted sample or standard were transferred into $1.5 \mathrm{~mL}$ Eppendorf Safe Lock tube. $800 \mu \mathrm{L}$ of anthrone reagent were added and homogenized with the sample solution through inversion. After $5 \mathrm{~min}$ of incubation on ice, the mixed solution was transferred into a thermo-incubator pre-heated at $95^{\circ} \mathrm{C}$ and shaken at $300 \mathrm{rpm}$ for 16 minutes and then cooled down on ice. Optical density of the cooled samples was measured at $625 \mathrm{~nm}$ and 
carbohydrate concentration was calculated using the standard curve and considering the dilution factors.

\subsection{Lipid extraction}

The lipid extraction protocol was fully described in (Silve et al., 2018). In brief, $5 \mathrm{~mL}$ of the microalgae suspension at $100 \mathrm{~g}$ Dw/L were centrifuged, supernatant was disregarded and the wet microalgae pellet was resuspended in a hexane-ethanol blend in order to reach a final extraction system of Water/Ethanol/Hexane, 1:18:7.3 vol/vol/vol. Extraction was performed overnight with agitation and in the dark. Then, Hexane and water were added in order to accomplish phase separation. The upper hexane phase was collected, and hexane evaporated under nitrogen flow. Extraction yields were determined gravimetrically in a precision balance.

\subsection{Evaluation of total lipid content}

The total lipid content was evaluated using freeze-dried biomass. The biomass was bead-milled (Mixer mill, MM400, Retsch, Haan, Germany) and then extracted in a Soxhlet apparatus (Behrotest Kompact-Apparatur KEX 30, Behr Labortechnik) using hexane as an extraction solvent. Details are given in (Silve et al., 2018). For each microalgae batch, lipid content was evaluated in triplicates.

\subsection{Experimental replication}

Experiments were replicated with three independently cultivated batches of microalgae culture. Results are presented as the average and standard deviation (std). To improve clarity of the Figures, only positive error bars (+std) are shown. Note that for figure 2 some conditions include only two repetitions as mention in the caption itself. 


\section{Results and discussion}

\subsection{Time course measurements of conductivity of microalgae suspension after PEF treatment}

One of the first consequences of the PEF-treatment on the microalgae is the release of intracellular ions which can be detected by measuring the conductivity of the microalgae suspension after treatment. Preliminary experiments, performed on mixotrophically grown microalgae, consisted in following the increase of conductivity of the suspension during the three hours after PEF-treatment with different specific energies. For all treatment conditions, the electric field was kept at $40 \mathrm{kV} / \mathrm{cm}$ and the pulse duration at $1 \mu \mathrm{s}$. The specific treatment energy was adjusted by selecting the appropriate pulse repetition rate, as described in Table 1 in the material and method section. Control suspension was pumped through the treatment chamber without applying any pulse. The results are displayed in Fig. 2 . The measured conductivity values were corrected for temperature impact by applying Eq. 7 and correspond to the reference temperature of $25^{\circ} \mathrm{C}$. The measurements indicate that the conductivity of untreated microalgae suspension was very stable over time. On the contrary, all PEF-treated samples display a conductivity jump just after the PEF-treatment and an additional conductivity increase at least during the three following hours. No full stabilization was reached. For the two highest specific energies tested, i.e. 100 and $150 \mathrm{~kJ} / \mathrm{L}$, the conductivities after PEF-treatment are very close at any time-point, suggesting that a maximum effect of PEF-treatment is reached for energies above $100 \mathrm{~kJ} / \mathrm{L}$. For all other energies tested, conductivity increase is lower but significant. Even the lowest tested energy, i.e. $5 \mathrm{~kJ} / \mathrm{L}$, induces a jump of conductivity from $1.1 \mathrm{mS} / \mathrm{cm}$ to $1.6 \mathrm{mS} / \mathrm{cm}$ immediately after PEF-treatment, and a further increase to about $2.4 \mathrm{mS} / \mathrm{cm}$ after 3 hours of waiting time. This experiment revealed that energy input as low as $5 \mathrm{~kJ} / \mathrm{L}$ could be significantly detected based on the conductivity of the microalgae suspension. The fact that conductivity of 
microalgae suspension continues to increase during at least the first three hours after the PEFtreatment suggest that the treatment causes long-term alteration of the microalgae cells. In the following section, experiments were performed to investigate if those long-term consequences could be used beneficially to improve spontaneous release of water-soluble compounds such as ions or carbohydrate as well as to increase the lipid yields after subsequent solvent extraction. In those experiments, the minimum energy was adjusted to $15 \mathrm{~kJ} / \mathrm{L}$. Moreover, for all the extraction experiments, the samples were covered with nitrogen and kept in the dark immediately after the PEF-treatment in order to avoid any degradation of molecules due to oxidation and photooxidation. The waiting time after the PEF-treatment is thereafter referred to as incubation time.

\subsection{Effect of PEF combined with incubation on the release of water fraction}

The effect of the PEF-treatment with different energy inputs on the spontaneous release of water soluble molecules was analyzed. Experiments were performed on both mixotrophically and autotrophically grown microalgae and always led to similar results. Only results from the autotrophically grown microalgae are reported since those are more representative for the biomass that will be used in industrial applications. After a given incubation time, the samples were centrifuged, the supernatant was collected and the carbohydrate content was quantified. Results for the different energies and for different duration of incubation are presented on Fig. 3a. The samples which were centrifuged immediately after PEF treatment and not incubated are represented on the graph at the time point 0.1 hour i.e. 6 minutes. For those samples, the concentration of carbohydrates in the supernatant depends highly on the treatment specific energy starting from $2.0 \pm 1.3 \mathrm{~g} / \mathrm{L}$ at $15 \mathrm{~kJ} / \mathrm{L}$, and reaching $8.3 \pm 1.5 \mathrm{~g} / \mathrm{L}$ at the highest tested energy of $150 \mathrm{~kJ} / \mathrm{L}$. For this last sample, the concentration of carbohydrates in the supernatant is not impacted by the duration of incubation. For all other tested energies, the amount of carbohydrate 
released increases with the duration of incubation. After only one hour of incubation, an energy of $50 \mathrm{~kJ} / \mathrm{L}$ leads to the same amount of carbohydrates release as the three-fold energy. After the longest tested incubation i.e. 20 hours, the level of carbohydrates released with $15 \mathrm{~kJ} / \mathrm{L}$ and $25 \mathrm{~kJ} / \mathrm{L}$ have reached $6.6 \pm 1.1$ and $8.2 \pm 1.1 \mathrm{~g} / \mathrm{L}$, i.e. 80 and $99 \%$ of the initial value obtained with $150 \mathrm{~kJ} / \mathrm{L}$. No release of carbohydrates was detected in the supernatant of the untreated suspension, for all tested incubation durations.

An experiment was designed in order to test whether the progressive release of carbohydrates after treatment could be blocked at low temperature. For that purpose, the incubation after treatment was performed either at the standard temperature of $25^{\circ} \mathrm{C}$ or on ice. Results for different treatment energies are displayed on Fig. 3b. As previously obtained, the amount of carbohydrates released after PEF-treatment with $150 \mathrm{~kJ} / \mathrm{L}$ is maximum directly after the treatment and does not increase further with incubation time. For all other energies, an increase of carbohydrates release with incubation time can be observed both at $25^{\circ} \mathrm{C}$ and on ice. However, the dynamics of the release of carbohydrates are slowed down on ice.

Moreover, the conductivity of the supernatant was analyzed. It provides an indirect measurement of the amount of charged molecules or ions spontaneously released. The results for incubation at $25^{\circ} \mathrm{C}$ are presented in Fig $4 \mathrm{a}$. For all tested PEF-treatment energies, the conductivity of the supernatant increases with the duration of incubation but with dynamics very different from the one of the carbohydrates. All samples tend to target the same value of $3500 \mu \mathrm{S} / \mathrm{cm}$ after 20 hours of incubation, but no stabilization has been observed at that time for any of the tested conditions, even not for the highest energy of $150 \mathrm{~kJ} / \mathrm{L}$, suggesting that ion release from the biomass still continues. During the first hours of incubation the conductivity of the supernatant of the control 
sample was stable around $1300 \mu \mathrm{S} / \mathrm{cm}$. Later it slightly increased and reached $1500 \mu \mathrm{S} / \mathrm{cm}$ after 20 hours of incubation.

The same measurements were performed on samples incubated on ice and the results are displayed on Fig. 4b. As obtained for the carbohydrates release, it can be observed that reducing the incubation temperature does not inhibit the increase of the conductivity of the supernatant. However, the rate of increase is reduced when samples are incubated on ice as compared to $25^{\circ} \mathrm{C}$.

\subsection{Effect of PEF treatment combined with incubation on lipid extraction yields}

The impact of PEF-treatment energy reduction coupled with incubation was also tested on lipid extraction. Samples were handled as described previously, i.e. PEF-treated and incubated in inert conditions. After incubation, samples were centrifuged to remove the water fraction and the microalgae pellet was resuspended in an ethanol-hexane mixture for lipid extraction. The lipid yields for different energies and different incubation times are displayed in Fig. 5a. The results indicate that incubation after PEF treatment is also beneficial for lipid extraction. The samples treated with the highest energy, i.e. $150 \mathrm{~kJ} / \mathrm{L}$, give a maximum yield after about two hours; the exact time was not determined. The amount of extracted lipids represents $90 \%$ of the evaluated total lipid content. For all other treatment energies, lipid yields are extremely low when extraction is performed immediately after the PEF-treatment but increase significantly with the incubation

duration. After 20 hours of incubation, the lipid yield from the samples treated with 15,25 and 50 $\mathrm{kJ} / \mathrm{L}$ have reached $76 \%, 91 \%$ and $97 \%$ of the values of samples treated with $150 \mathrm{~kJ} / \mathrm{L}$.

As for the release of water fraction, the effect of incubation on ice was tested. Additionally, some samples were centrifuged just after PEF treatment and left for incubation in the pellet form at 
$25^{\circ} \mathrm{C}$ in inert conditions. Results are displayed on Fig. 5b. As previously, incubating the samples on ice rather than at $25^{\circ} \mathrm{C}$ did not completely suppress the increase of lipid yield with incubation time, however, the effect is considerably slowed down. Incubation in the pellet form also had a small inhibiting effect as compared to incubation in the suspension form but this effect was much less pronounced than the effect of reduction of temperature.

\section{4. Correlation between water soluble release and lipid extraction}

In this study, lipid extraction was performed with a monophasic solvent system which requires penetration of the solvent inside the cells. One of the questions raised by the results is whether solvent penetration and subsequent lipid extraction are facilitated by the modifications induced by the PEF treatment on the membrane and eventually on the cell-wall or whether the release of the water-soluble molecules modifies the properties of the intracellular space and therefore enables a more efficient solvent intracellular penetration. A first experimental attempt to answer this question consisted in centrifuging the microalgae suspension just after PEF-treatment, removing the supernatant, and performing incubation of the resulting microalgae pellet before lipid extraction (Fig. 5). Indeed, in the pellet form it can be expected that release of intracellular components during incubation is limited, since the volume available for extracellular diffusion is comparatively small. Additionally, in the case of pellet incubation, released carbohydrates or ions are still present in the extraction system in contrast to alternatively incubating the complete suspension and centrifuging it just before lipid extraction. In the latter case a major part of the water-soluble ions and molecules is removed prior to the solvent extraction step. Incubating the pellet only was indeed less efficient than incubating the microalgae suspension and, consistently, 
longer incubation times were required to reach the same lipid yields. This suggests a correlation between the release of water soluble molecules and the ability to extract lipids. The correlation between the different extraction yields can be further highlighted by the graphs on Fig. 6, which represent dot plots of lipids yield and carbohydrate release, respectively, versus conductivity of supernatants. Each marker represents a single sample. Data points with the same shape of markers correspond to one global experiment. Empty markers in blue correspond to samples incubated on ice. Samples for all tested PEF-treatment energies and all incubation times have been included. One can see that the samples incubated on ice follow the same behavior as the other samples. A general trend line along all data points can be observed despite some scattering of the data. One can see that the release of the carbohydrates starts as soon as the conductivity of the supernatant increases, Fig. 6A. On the contrary, the lipid yield is not improved unless the conductivity of the supernatant has reached about 2000-2200 $\mu \mathrm{S} / \mathrm{cm}$, Fig. $6 \mathrm{~B}$. It therefore appears as if the release of ions and carbohydrates is the earliest event. The modifications that enable lipid extraction are later events which might be some consequences of the earlier release (Parniakov et al., 2015) or might be independent. The question remains to be addressed and more generally the mechanism by which PEF-treatment facilitates extractions should be investigated.

\subsection{Nature of the effect induces by PEF-treatment}

PEF-treatment is always associated with a temperature increase of the treated suspension induced by Joule effect. However, the maximum temperature elevation evaluated assuming adiabatic conditions i.e. worst case scenario, was of $36^{\circ} \mathrm{C}$ for the highest treatment energy i.e. $150 \mathrm{~kJ} / \mathrm{L}$. In practice, as previously reported (Silve et al., 2018), the measured temperature at the 
output of the treatment chamber was close to $46^{\circ} \mathrm{C}$, which corresponds to an elevation of about $26^{\circ} \mathrm{C}$. Additionally, for the low treatment energies, such as 15 or $25 \mathrm{~kJ} / \mathrm{L}$, the temperature elevation is only of a few degrees (Table 1 ). It can therefore be excluded that temperature elevation is the reason for water-fraction release and facilitated lipid extraction.

Waiting after applying PEF-treatment was already suggested previously (Coustets et al., 2015, 2013; Goettel et al., 2013) based on the fact that release of molecules is a diffusion based process. In this paper, it was shown that water carbohydrates continue to be released after $20 \mathrm{~h}$.

Additionally, the dynamics of the increase of conductivity of the supernatant follow a convex increasing curve when displayed in the log scale. These elements suggest, therefore, that more than just diffusion is at play and the mechanisms responsible for the observed effect remain to be explained. It is already known that a cascade of events is induced after PEF (Straessner et al., 2013), most probably leading to cell death. Moreover, in this study, incubation was performed in the dark and the airspace in the vial over the sample was displaced with nitrogen. This was initially intended to avoid oxidation of various molecules, especially of lipids, but from a biological point of view, those anoxic conditions can rush cell-death. The effects of incubation are in all cases slowed down when incubation is performed on ice. The possible role of a contamination was considered since at the harvesting step and onwards, experiments were not performed in sterile conditions anymore. This could be compatible with the reduction of the effect which is observed when incubation is done on ice since bacterial growth will be reduced at such low temperature. This was not directly tested but observation of biomass under the microscope after 20 hours of incubation did not indicate contaminations. Another plausible effect is that enzymes of the microalgae start to degrade the biomass. A degradation of cells under the action of their endogenous enzymes has been already well described for yeasts and is referred to as autolysis (Alexandre and Guilloux- 
Benatier, 2006; Babayan and Bezrukov, 1985; Hernawan and Fleet, 1995). It is characterized by loss of membrane function, hydrolysis of intracellular polymers and consequently release of the hydrolytic products in the extracellular space (Alexandre and Guilloux-Benatier, 2006; Hernawan and Fleet, 1995). Most important, autolysis in yeast has been shown to induces cell-wall degradation without full break-down. If a similar degradation of the microalgae cell-wall was taking place after PEF-treatment, this could partially explain facilitated solvent penetration and therefore lipid extraction.

On yeast, natural autolysis is described as a relatively slow process extending over days or weeks. Nevertheless, it can be accelerated by some external inductors such as some chemicals. Typical duration of induced autolysis, as performed for example in some wine industries, range from $48 \mathrm{~h}$ to $72 \mathrm{~h}$ (Alexandre and Guilloux-Benatier, 2006). In the case of PEF-treatment of microalgae cells, some effects are observed in the next minutes following the treatment and can be most probably attributed to some direct effect i.e. permeabilisation of the membrane followed by diffusion of small permeant molecules. The more delayed effects which extent over at least $20 \mathrm{~h}$ as seen from the conductivity of the supernatant (Fig. 4) could however be explained by an autolysis-like process accelerated by permeabilisation of the plasma membrane and eventually of intracellular membrane leading to the intracellular release of still functional enzymes. In fact, PEF-treatment was already suggested as an autolysis inducer in the yeast Saccharomyces cerevisiae (Martínez et al., 2016). Future work should focus on elucidating the possible role of an autolysis-like process. In particular, supernatant should be checked for autolysis products such as free amino-acids, sugar monomer or organic acids. Additionally, it should be investigated whether a natural autolysis of microalgae cells induced for example by starvation could induce the same effects as PEFtreatment especially on lipid extraction. Note that in a recent published study on the effect of PEF- 
treatment on Chlorella vulgaris, very different results were reported since time course measurements of conductivity after PEF-treatment showed stabilization after about $1 \mathrm{~h}$ (Carullo et al., 2018). The longer pulses used in this study, which are less prone to induce intracellular damages might partially explain the observed differences.

\subsection{Practical applications}

The long-term consequences observed in this study could be used beneficially to improve spontaneous release of water-soluble compounds such as ions or carbohydrate as well as to increase the lipid yields after subsequent solvent extraction. Indeed, adding an incubation time after PEF-treatment considerably increased all extraction yields and enables in turn to reduce the energy input of the PEF-treatment. Previous work, had shown that for autotrophically grown Auxenochlorella protothecoides, and with similar extraction procedure, $90 \%$ of the evaluated total lipid content could be extracted after PEF-treatment with $150 \mathrm{~kJ} / \mathrm{L}$ translating into $1.5 \mathrm{MJ} / \mathrm{kg}$ DW. In this previous work, the effects of incubation were however unknown and microalgae suspension were kept on ice after PEF-treatment before being processed. This current study, demonstrates that with a long incubation time, e.g. 20 hours, energy inputs of 15, 25 and $50 \mathrm{~kJ} / \mathrm{L}$ are enough to extract $68.8 \%, 82.2 \%$ and $87.5 \%$ of the evaluated total lipid content. Concerning the carbohydrates, their extraction was not the primary goal of the study. Nevertheless, the spontaneous releases that occur can be beneficial for applications focusing on carbohydrates release or in a bio-refinery concept aiming at multicomponent valorization. Note that in order to maximize the recovery of the carbohydrates a washing step of the pellet should be performed in order to recover the carbohydrates trapped in the pellet. This was not included in this study and therefore no absolute recovery percentages have been calculated since those would be under evaluating the full potential of the method. Additionally, recovery of carbohydrate can only be 
considered in case those molecules are not hydrolyzed by endogenous enzymes as discussed previously.

The reduction of energy requirements which is enabled by the incubation step could become very valuable for applications in which energy consumption is a critical factor. In principle, it might be possible to even further reduce the PEF-treatment energy by acting on other parameters. Increasing the concentration of the microalgae suspension before applying PEF-treatment might for example lead to a further reduction of the overall energy requirement. Up to this point, a reduction of the energy demand by a factor of 6 without significant changes in lipid or carbohydrate yield could be demonstrated in this study by including an incubation step into the PEF-processing scheme. For A.p, processed at a biomass density of $100 \mathrm{~g}_{\mathrm{DW}} / \mathrm{L}, \mathrm{PEF}$ treatment energy requirements are reduced from $1.5 \mathrm{MJ} / \mathrm{kg}_{\text {DW }}$ to $0.25 \mathrm{MJ} / \mathrm{kg}_{\mathrm{DW}}$ Additionally, a reduction of the electric field intensity or of the pulse duration might further reduce the energy demand. This was not investigated in this study so far, but can be addressed in future.

\section{Conclusion}

Incubation after PEF-treatment of Auxenochlorella protothecoides was shown to strongly improve the efficiency of extraction of water-soluble components such as ions or carbohydrate as well as lipid yields from subsequent solvent extraction. Consequently, PEF-treatment specific energy can be reduced down to $0.25 \mathrm{MJ} / \mathrm{kg}_{\mathrm{DW}}$ when coupled with 20 hour incubation at $25^{\circ} \mathrm{C}$ and still leads to high extraction yields. By allowing significant reduction of operating costs, this strategy has high potential benefit for industrial applications. In the future, understanding how the biomass 
evolution during incubation facilitates extractions processes will enable further optimization of the whole microalgae down-stream processing.

\section{Funding source}

This work was conducted in the framework and financed by the Helmholtz Research Program on Renewable Energies [Topic 3: Bioenergy] and by the European Union's Horizon 2020 Research and Innovation program [Grant Agreement No. 727874]

\section{Declaration of interest}

none 


\section{Reference}

1 Alexandre, H., Guilloux-Benatier, M., 2006. Yeast autolysis in sparkling wine - a review. Aust. J. Grape Wine Res. 12, 119-127. https://doi.org/10.1111/j.1755-0238.2006.tb00051.x

2 Azencott, H.R., Peter, G.F., Prausnitz, M.R., 2007. Influence of the Cell Wall on Intracellular Delivery to Algal Cells by Electroporation and Sonication. Ultrasound Med. Biol. 33, 1805-1817. https://doi.org/10.1016/j.ultrasmedbio.2007.05.008

3 Babayan, T.L., Bezrukov, M.G., 1985. Autolysis in yeasts. Acta Biotechnol. 5, 129-136. https://doi.org/10.1002/abio.370050205

4 Bodénès, P., Lopes, F., Pareau, D., Français, O., Le Pioufle, B., 2016. Microdevice for studying the in situ permeabilization and characterization of Chlamydomonas reinhardtii in lipid accumulation phase. Algal Res. 16, 357-367. https://doi.org/10.1016/j.algal.2016.03.023

5 Carullo, D., Abera, B.D., Casazza, A.A., Donsì, F., Perego, P., Ferrari, G., Pataro, G., 2018. Effect of pulsed electric fields and high pressure homogenization on the aqueous extraction of intracellular compounds from the microalgae Chlorella vulgaris. Algal Res. 31, 60-69. https://doi.org/10.1016/j.algal.2018.01.017

6 Chew, K.W., Yap, J.Y., Show, P.L., Suan, N.H., Juan, J.C., Ling, T.C., Lee, D.-J., Chang, J.-S., 2017. Microalgae biorefinery: High value products perspectives. Bioresour. Technol. 229, 53-62. https://doi.org/10.1016/j.biortech.2017.01.006

7 Cooney, M.J., Young, G., Pate, R., 2011. Bio-oil from photosynthetic microalgae: Case study. Bioresour. Technol., Special Issue: Biofuels - II: Algal Biofuels and Microbial Fuel Cells 102, 166177. https://doi.org/10.1016/j.biortech.2010.06.134

8 Coustets, M., Al-Karablieh, N., Thomsen, C., Teissié, J., 2013. Flow Process for Electroextraction of Total Proteins from Microalgae. J. Membr. Biol. 246, 751-760. https://doi.org/10.1007/s00232013-9542-y

9 Coustets, M., Joubert-Durigneux, V., Hérault, J., Schoefs, B., Blanckaert, V., Garnier, J.-P., Teissié, J., 2015. Optimization of protein electroextraction from microalgae by a flow process.

Bioelectrochemistry, BIOELECTRICS 2013 103, 74-81. https://doi.org/10.1016/j.bioelechem.2014.08.022

10 Eing, C., Goettel, M., Straessner, R., Gusbeth, C., Frey, W., 2013. Pulsed Electric Field Treatment of Microalgae -Benefits for Microalgae Biomass Processing. IEEE Trans. Plasma Sci. 41, 2901-2907. https://doi.org/10.1109/TPS.2013.2274805

11 Frey, W., Gusbeth, C., Sakugawa, T., Sack, M., Mueller, G., Sigler, J., Vorobiev, E., Lebovka, N., Álvarez, I., Raso, J., Heller, L.C., Malik, M.A., Eing, C., Teissie, J., 2017. Environmental Applications, Food and Biomass Processing by Pulsed Electric Fields, in: Bioelectrics. Springer, Tokyo, pp. 389-476. https://doi.org/10.1007/978-4-431-56095-1_6

12 Goettel, M., Eing, C., Gusbeth, C., Straessner, R., Frey, W., 2013. Pulsed electric field assisted extraction of intracellular valuables from microalgae. Algal Res. 2, 401-408. https://doi.org/10.1016/j.algal.2013.07.004

13 Golberg, A., Sack, M., Teissie, J., Pataro, G., Pliquett, U., Saulis, G., Stefan, T., Miklavcic, D., Vorobiev, E., Frey, W., 2016. Energy-efficient biomass processing with pulsed electric fields for bioeconomy and sustainable development. Biotechnol. Biofuels 9, 94.

https://doi.org/10.1186/s13068-016-0508-z 
14 Grimi, N., Dubois, A., Marchal, L., Jubeau, S., Lebovka, N.I., Vorobiev, E., 2014. Selective extraction from microalgae Nannochloropsis sp. using different methods of cell disruption. Bioresour. Technol. 153, 254-259. https://doi.org/10.1016/j.biortech.2013.12.011

15 Grimnes, S., Martinsen, Ø.G., 2008. Bioimpedance and bioelectricity basics. Academic Press.

16 Günerken, E., D’Hondt, E., Eppink, M.H.M., Garcia-Gonzalez, L., Elst, K., Wijffels, R.H., 2015. Cell disruption for microalgae biorefineries. Biotechnol. Adv. 33, 243-260. https://doi.org/10.1016/j.biotechadv.2015.01.008

17 Hernawan, T., Fleet, G., 1995. Chemical and cytological changes during the autolysis of yeasts. J. Ind. Microbiol. 14, 440-450. https://doi.org/10.1007/BF01573955

18 Kempkes, M.A., 2016. Pulsed Electric Fields for Algal Extraction and Predator Control, in: Handbook of Electroporation. Springer, Cham, pp. 1-16. https://doi.org/10.1007/978-3-319-26779-1_2151

19 Lai, Y.S., Parameswaran, P., Li, A., Baez, M., Rittmann, B.E., 2014. Effects of pulsed electric field treatment on enhancing lipid recovery from the microalga, Scenedesmus. Bioresour. Technol. 173, 457-461. https://doi.org/10.1016/j.biortech.2014.09.124

20 Levine, Z.A., Vernier, P.T., 2010. Life cycle of an electropore: field-dependent and fieldindependent steps in pore creation and annihilation. J. Membr. Biol. 236, 27-36. https://doi.org/10.1007/s00232-010-9277-y

21 Luengo, E., Martínez, J.M., Coustets, M., Álvarez, I., Teissié, J., Rols, M.-P., Raso, J., 2015. A Comparative Study on the Effects of Millisecond- and Microsecond-Pulsed Electric Field Treatments on the Permeabilization and Extraction of Pigments from Chlorella vulgaris. J. Membr. Biol. 248, 883-891. https://doi.org/10.1007/s00232-015-9796-7

22 Martínez, J.M., Cebrián, G., Álvarez, I., Raso, J., 2016. Release of Mannoproteins during Saccharomyces cerevisiae Autolysis Induced by Pulsed Electric Field. Front. Microbiol. 7. https://doi.org/10.3389/fmicb.2016.01435

23 Mir, L.M., 2008. Application of Electroporation Gene Therapy: Past, Current, and Future, in: Electroporation Protocols, Methods in Molecular Biology ${ }^{\mathrm{TM}}$. Humana Press, pp. 3-17. https://doi.org/10.1007/978-1-59745-194-9_1

$24 \mathrm{Mir}$, L.M., 2006. Bases and rationale of the electrochemotherapy. Eur. J. Cancer Suppl., Electrochemotherapy 4, 38-44. https://doi.org/10.1016/j.ejcsup.2006.08.005

25 Olmstead, I.L.D., Kentish, S.E., Scales, P.J., Martin, G.J.O., 2013. Low solvent, low temperature method for extracting biodiesel lipids from concentrated microalgal biomass. Bioresour. Technol. 148, 615-619. https://doi.org/10.1016/j.biortech.2013.09.022

26 Pakhomov, A.G., Miklavčič, D., Markov, M.S., 2010. Advanced Electroporation Techniques in Biology and Medicine. CRC Press.

27 Parniakov, O., Barba, F.J., Grimi, N., Marchal, L., Jubeau, S., Lebovka, N., Vorobiev, E., 2015. Pulsed electric field assisted extraction of nutritionally valuable compounds from microalgae Nannochloropsis spp. using the binary mixture of organic solvents and water. Innov. Food Sci. Emerg. Technol. 27, 79-85. https://doi.org/10.1016/j.ifset.2014.11.002

28 Phong, W.N., Show, P.L., Ling, T.C., Juan, J.C., Ng, E.-P., Chang, J.-S., 2018. Mild cell disruption methods for bio-functional proteins recovery from microalgae-Recent developments and future perspectives. Algal Res. 31, 506-516. https://doi.org/10.1016/j.algal.2017.04.005

29 Poignard, C., Silve, A., Wegner, L., 2016. Different Approaches Used in Modeling of Cell Membrane Electroporation, in: Miklavcic, D. (Ed.), Handbook of Electroporation. Springer International Publishing, pp. 1-24. https://doi.org/10.1007/978-3-319-26779-1_3-1 
30 Posten, C., Walter, C., 2012. Microalgal Biotechnology: Integration and Economy. De Gruyter, Berlin, Boston. https://doi.org/10.1515/9783110298321

31 Postma, P.R., Miron, T.L., Olivieri, G., Barbosa, M.J., Wijffels, R.H., Eppink, M.H.M., 2015. Mild disintegration of the green microalgae Chlorella vulgaris using bead milling. Bioresour. Technol., Advances in biofuels and chemicals from algae 184, 297-304. https://doi.org/10.1016/j.biortech.2014.09.033

32 Postma, P.R., Pataro, G., Capitoli, M., Barbosa, M.J., Wijffels, R.H., Eppink, M.H.M., Olivieri, G., Ferrari, G., 2016. Selective extraction of intracellular components from the microalga Chlorella vulgaris by combined pulsed electric field-temperature treatment. Bioresour. Technol. 203, 80-88. https://doi.org/10.1016/j.biortech.2015.12.012

33 Postma, P.R., Suarez-Garcia, E., Safi, C., Yonathan, K., Olivieri, G., Barbosa, M.J., Wijffels, R.H., Eppink, M.H.M., 2017. Energy efficient bead milling of microalgae: Effect of bead size on disintegration and release of proteins and carbohydrates. Bioresour. Technol. 224, 670-679. https://doi.org/10.1016/j.biortech.2016.11.071

34 Sengel, J.T., Wallace, M.I., 2016. Imaging the dynamics of individual electropores. Proc. Natl. Acad. Sci. 113, 5281-5286. https://doi.org/10.1073/pnas.1517437113

35 Sheng, J., Vannela, R., Rittmann, B.E., 2011. Evaluation of Cell-Disruption Effects of Pulsed-ElectricField Treatment of Synechocystis PCC 6803. Environ. Sci. Technol. 45, 3795-3802. https://doi.org/10.1021/es103339x

36 Silve, A., Papachristou, I., Wüstner, R., Sträßner, R., Schirmer, M., Leber, K., Guo, B., Interrante, L., Posten, C., Frey, W., 2018. Extraction of lipids from wet microalga Auxenochlorella protothecoides using pulsed electric field treatment and ethanol-hexane blends. Algal Res. 29, 212-222. https://doi.org/10.1016/j.algal.2017.11.016

37 Spiden, E.M., Yap, B.H.J., Hill, D.R.A., Kentish, S.E., Scales, P.J., Martin, G.J.O., 2013. Quantitative evaluation of the ease of rupture of industrially promising microalgae by high pressure homogenization. Bioresour. Technol. 140, 165-171. https://doi.org/10.1016/j.biortech.2013.04.074

38 Straessner, R., Eing, C., Goettel, M., Gusbeth, C., Frey, W., 2013. Monitoring of Pulsed Electric Field-Induced Abiotic Stress on Microalgae by Chlorophyll Fluorescence Diagnostic. IEEE Trans. Plasma Sci. 41, 2951-2958. https://doi.org/10.1109/TPS.2013.2281082

39 't Lam, G.P., van der Kolk, J.A., Chordia, A., Vermuë, M.H., Olivieri, G., Eppink, M.H.M., Wijffels, R.H., 2017. Mild and Selective Protein Release of Cell Wall Deficient Microalgae with Pulsed Electric Field. ACS Sustain. Chem. Eng. 5, 6046-6053. https://doi.org/10.1021/acssuschemeng.7b00892

40 Tarek, M., 2005. Membrane electroporation: a molecular dynamics simulation. Biophys. J. 88, 4045-4053. https://doi.org/10.1529/biophysj.104.050617

41 Teissie, J., Golzio, M., Rols, M.P., 2005. Mechanisms of cell membrane electropermeabilization: A minireview of our present (lack of ?) knowledge. Biochim. Biophys. Acta BBA - Gen. Subj., Some Insights into the Biophysics of Complex SystemsInternational Biophysics Congress 1724, 270280. https://doi.org/10.1016/j.bbagen.2005.05.006

42 Toepfl, S., Heinz, V., Knorr, D., 2006. Applications of Pulsed Electric Fields Technology for the Food Industry, in: Pulsed Electric Fields Technology for the Food Industry, Food Engineering Series. Springer, Boston, MA, pp. 197-221. https://doi.org/10.1007/978-0-387-31122-7_7

43 Vanthoor-Koopmans, M., Wijffels, R.H., Barbosa, M.J., Eppink, M.H.M., 2013. Biorefinery of microalgae for food and fuel. Bioresour. Technol., Biorefineries 135, 142-149. https://doi.org/10.1016/j.biortech.2012.10.135 
44 Yap, B.H.J., Dumsday, G.J., Scales, P.J., Martin, G.J.O., 2015. Energy evaluation of algal cell disruption by high pressure homogenisation. Bioresour. Technol., Advances in biofuels and chemicals from algae 184, 280-285. https://doi.org/10.1016/j.biortech.2014.11.049

45 Yarmush, M.L., Golberg, A., Serša, G., Kotnik, T., Miklavčič, D., 2014. Electroporation-based technologies for medicine: principles, applications, and challenges. Annu. Rev. Biomed. Eng. 16, 295-320. https://doi.org/10.1146/annurev-bioeng-071813-104622

46 Zbinden, M.D.A., Sturm, B.S.M., Nord, R.D., Carey, W.J., Moore, D., Shinogle, H., Stagg-Williams, S.M., 2013. Pulsed electric field (PEF) as an intensification pretreatment for greener solvent lipid extraction from microalgae. Biotechnol. Bioeng. 110, 1605-1615.

https://doi.org/10.1002/bit.24829 
Table $1:$ Impact of the repetition rate on the average number of pulses delivered per volume unit and on the specific treatment energy. The specific treatment energy per kilogram of dry weight assumes a microalgae concentration of $10 \%[\mathrm{w} / \mathrm{w}]$ in the suspension.

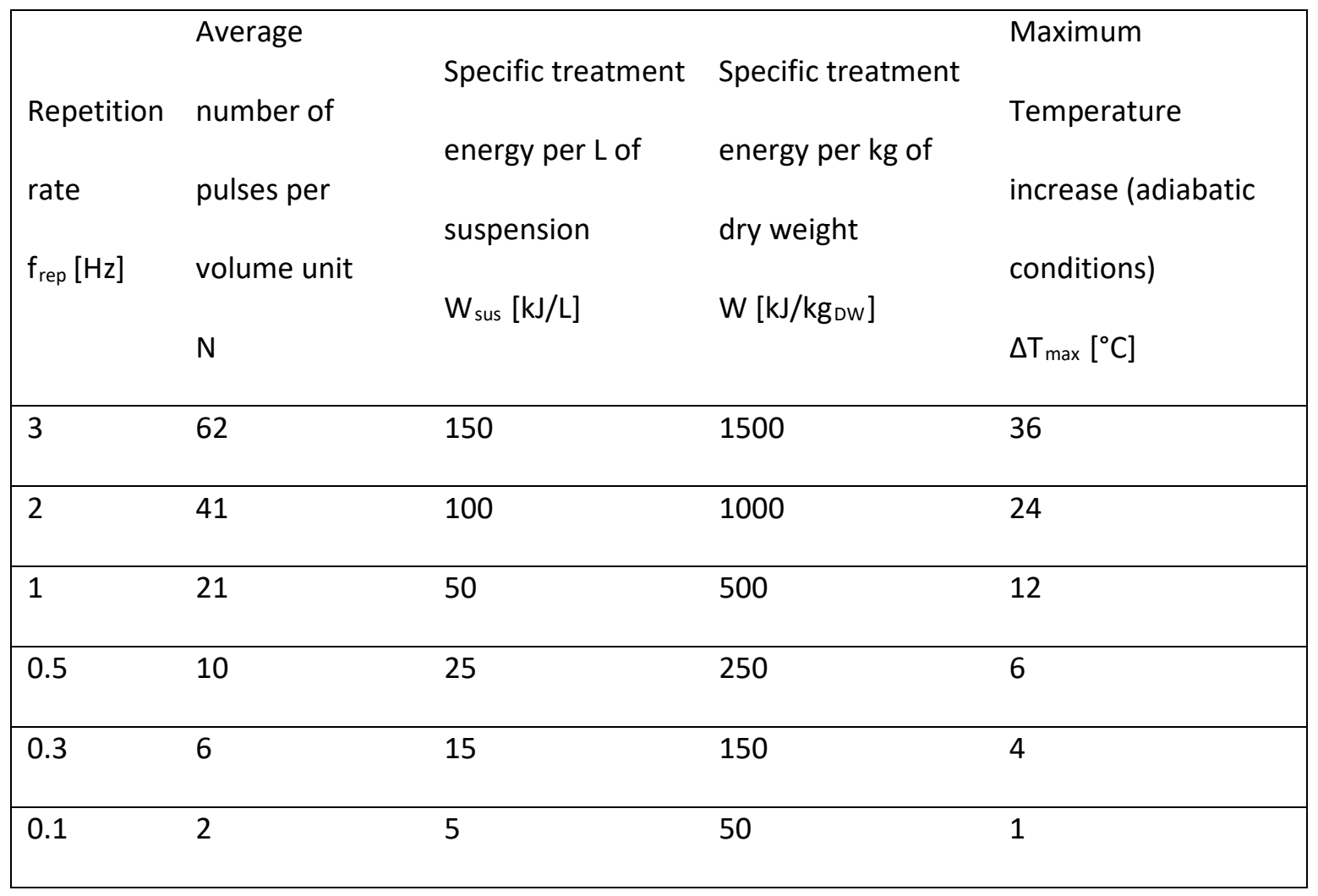




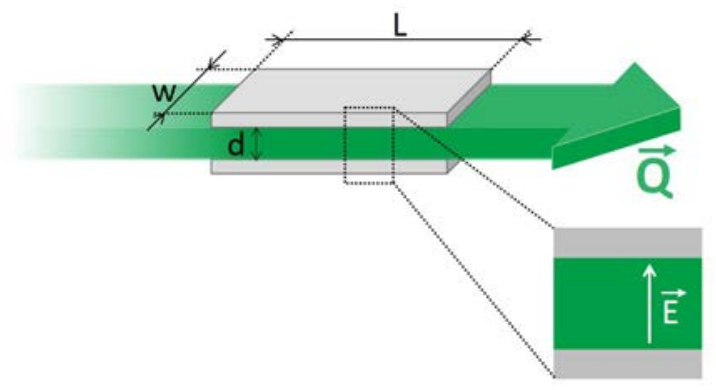

Fig. 1: Schematic representation of a PEF-treatment chamber operating in continuous flow mode.

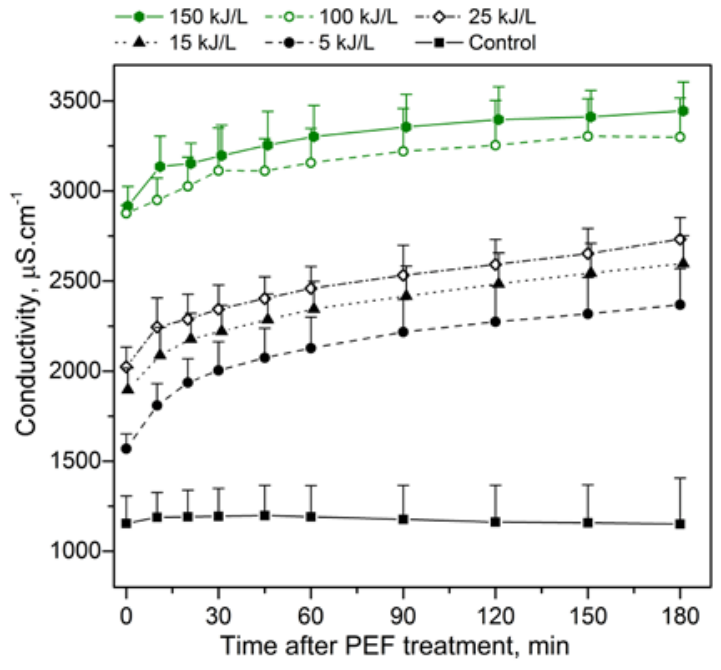

Fig. 2: Time course measurements of conductivity after PEF-treatment of microalgae suspensions with different specific treatment energies. Time 0 corresponds to the end of the treatment. The magnitude of the electric field was $40 \mathrm{kV} / \mathrm{cm}$, and the pulse duration $1 \mu \mathrm{s}$ in all experiment. Specific treatment energy was adjusted by selecting the appropriate repetition rate, cf. Table 1 . All conductivity data were correlated to the reference temperature of $25^{\circ} \mathrm{C}$. The control suspension, filled squares, was pumped through the treatment chamber without application of pulses. Results are the average + std of 2 to 3 independent experiments. 

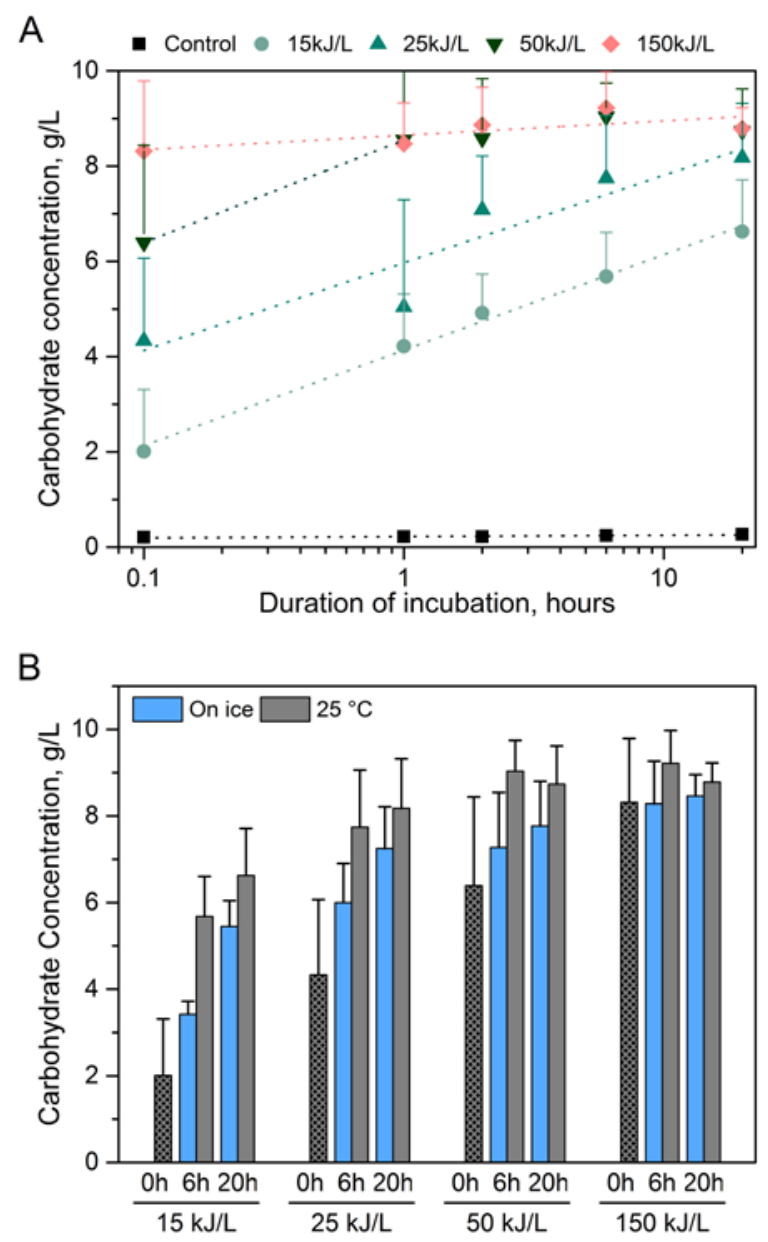

Fig. 3: concentration of carbohydrate spontaneously released in the supernatant (A) as a function of the duration of incubation after PEF-treatment and for different PEF treatment specific energy (B) immediately after PEF-treatment or after $6 \mathrm{~h}$ and $20 \mathrm{~h}$ of incubation, for different PEF treatment specific energy. Incubation was performed in an inert atmosphere with samples covered with nitrogen, and stored in the dark at $25^{\circ} \mathrm{C}(\mathrm{A}$ and $\mathrm{B}$ ) or on ice (B). For each sample, time zero corresponds to the end of the PEF-treatment. The control suspension was pumped through the treatment chamber without application of pulses. Results are the average + std of 3 independent experiments. Note that the data obtained at $25^{\circ} \mathrm{C}$ are the same in both panels $\mathrm{A}$ and $\mathrm{B}$. 

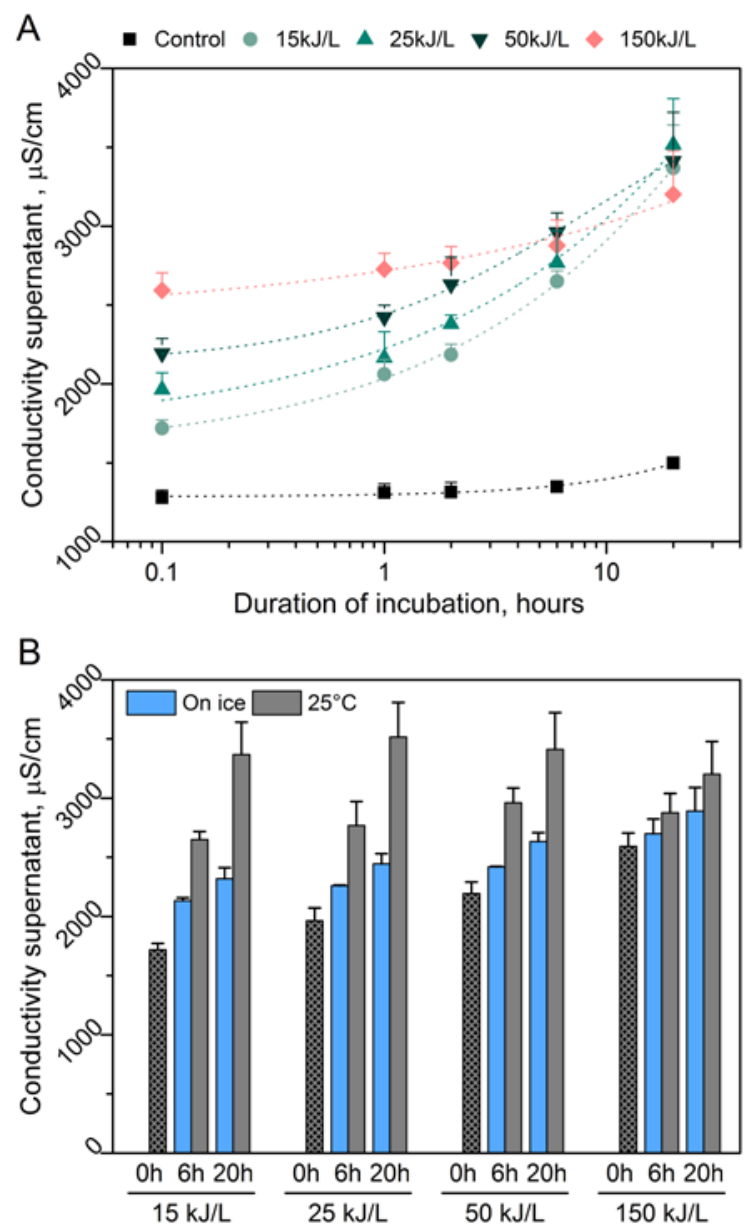

Fig. 4: Conductivity of the supernatant $(\mathrm{A})$ as a function of the duration of incubation after PEFtreatment and for different specific PEF treatment energies (B) immediately after PEF-treatment or after $6 \mathrm{~h}$ and $20 \mathrm{~h}$ of incubation, for different specific PEF treatment energies. Incubation was performed in an inert atmosphere with samples covered with nitrogen, and stored in the dark at $25^{\circ} \mathrm{C}(\mathrm{A}$ and $\mathrm{B}$ ) or on ice (B). For each sample, time zero corresponds to the end of the PEFtreatment. The control suspension was pumped through the treatment chamber without application of pulses. The displayed data correspond to the calculated conductivity value at $25^{\circ} \mathrm{C}$. Results are the average + std of 3 independent experiments. Note that the data obtained at $25^{\circ} \mathrm{C}$ are the same in both panels $A$ and $B$. 

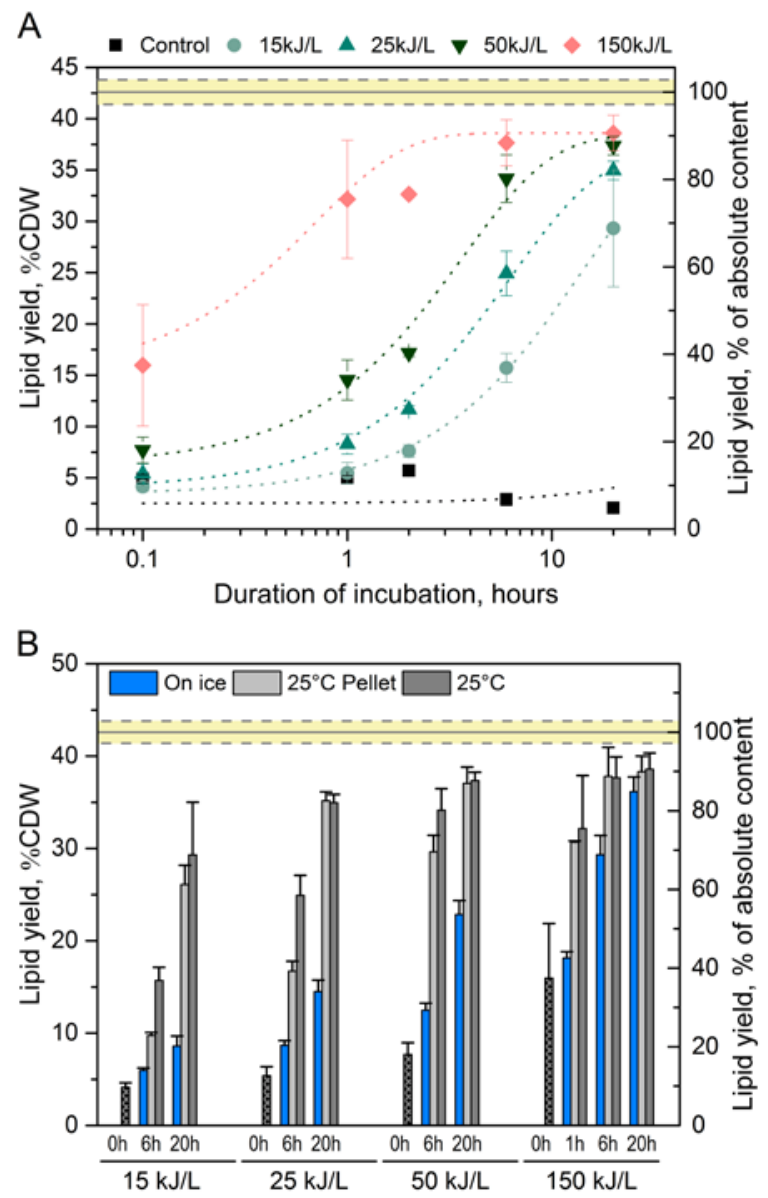

Fig. 5: Lipid extraction yield (A) as a function of the duration of incubation after PEF-treatment and for different specific PEF treatment energies (B) immediately after PEF-treatment or after $1 \mathrm{~h}, 6 \mathrm{~h}$ and 20h of incubation, for different PEF treatment specific energy. Incubation was performed in an inert atmosphere with samples covered with nitrogen, and stored in the dark at $25^{\circ} \mathrm{C}(\mathrm{A}$ and $\mathrm{B})$ or on ice (B). The samples marked as ' $25^{\circ} \mathrm{C}$ Pellet' correspond to samples which were centrifuged just after PEF-treatment and incubated in pellet form rather that incubating the initial microalgae suspension. For each sample, time zero corresponds to the end of the PEF-treatment. Results are the average + std of 3 independent experiments. The yellow line corresponds to the evaluated 
absolute lipid content (average \pm std). Note that the data obtained at $25^{\circ} \mathrm{C}$ are the same in both panels $\mathrm{A}$ and $\mathrm{B}$.
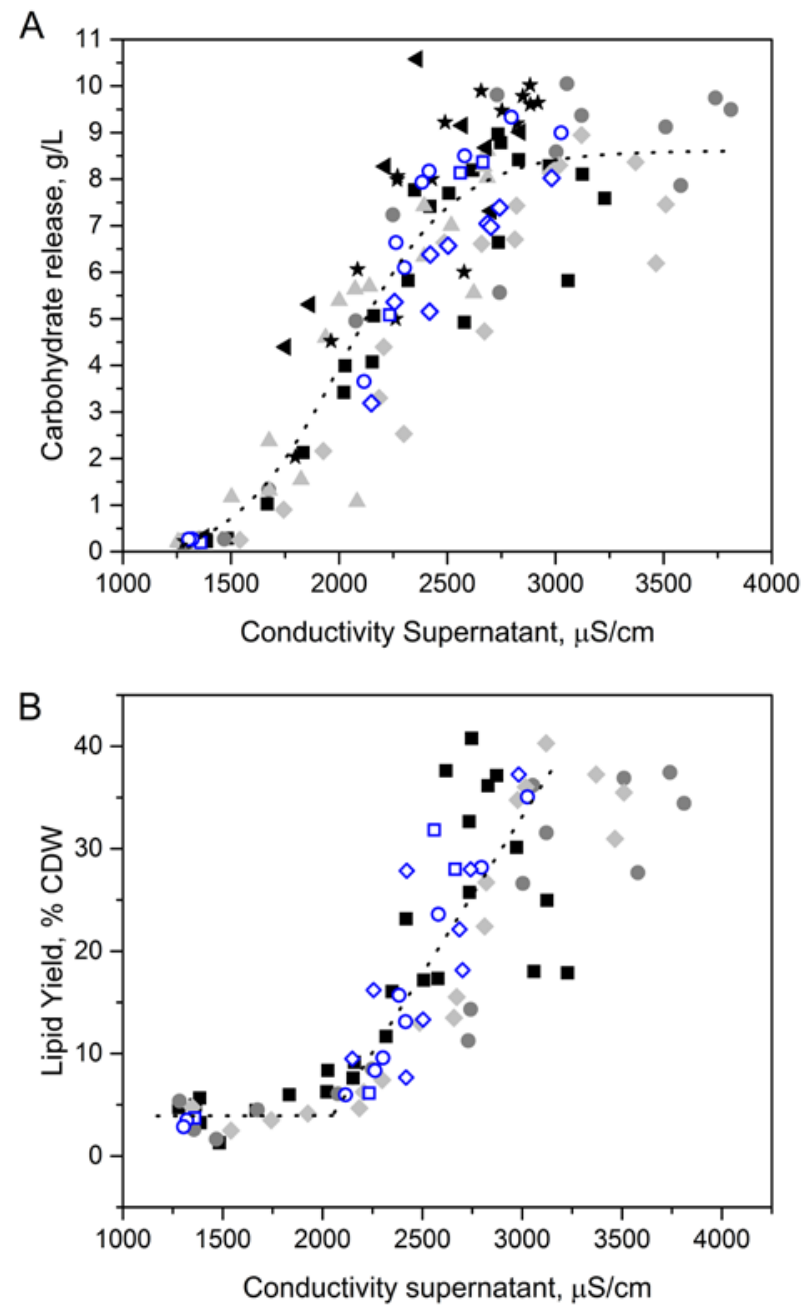

Fig. 6: correlation between (A) carbohydrate release and conductivity of supernatant, (B) Lipid yield and conductivity of supernatant. Each marker corresponds to an individual sample. One marker shape (square, circle, diamond etc...) correspond to one set of experiments. The full markers are data points of samples incubated at $25^{\circ} \mathrm{C}$ while the empty blue markers are results from samples incubated on ice. 